\title{
Angular dispersion of protons transmitted through thin gold films
}

\author{
C. Archubi ${ }^{* 1,}$, C. Denton ${ }^{2}$, J. C. Eckardt ${ }^{1}$, G. H. Lantschner ${ }^{1}$, F. Lovey ${ }^{1}$, J. Valdés ${ }^{2,3}$, \\ C. Parra ${ }^{2}$, F. Zappa ${ }^{4}$, and N. R. Arista ${ }^{1}$ \\ ${ }^{1}$ División Colisiones Atómicas, Centro Atómico Bariloche, Instituto Balseiro, S.C. de Bariloche, \\ Argentina \\ 2 Departamento de Física, Universidad Técnica Federico Santa María, Casilla 110-V, Valparaíso, Chile \\ ${ }^{3}$ Departamento de Física, Universidad de Santiago de Chile, Casilla 307, Santiago, Chile \\ ${ }^{4}$ Universidade Federal do Rio de Janeiro, Rio de Janeiro, Brasil
}

Received 16 December 2003, revised 21 May 2004, accepted 24 May 2004

Published online 13 July 2004

PACS 07.05.Tp, 34.20.Cf, 34.50.Lf, 79.20.Rf

The angular distributions of protons travelling through thin polycrystalline gold targets $(\sim 15 \mathrm{~nm})$ with incident energies in the range of $4-10 \mathrm{keV}$ have been measured. The results confirm previous experiments at higher energies showing deviations from theoretical predictions based on the standard multiple scattering theory. In order to prove that the effect of crystal structure is one of the main causes of these deviations we have performed numerical simulations. To simulate the polycrystalline structure in a realistic way, we have made an analysis of the target by means of transmission electron microscopy (TEM) techniques. Including these characteristics in the simulation, together with the effect of vibrations and crystal disorder we analyzed the corresponding angular distribution. To evaluate the role of channeling, we also measured angular distributions of protons in a $\langle 100\rangle$ gold foil and made the corresponding numerical simulations. The results show the critical influence of the target structure in the angular spectra of transmitted ions.

(C) 2004 WILEY-VCH Verlag GmbH \& Co. KGaA, Weinheim

\section{Introduction}

During the last thirty years a large number of experimental results have been published involving multiple scattering of protons in thin films. Most of these studies have been made using amorphous and polycrystalline materials. The interpretation of the measurements has been developed following a theoretical approach based on Meyer's [1] and Sigmund's [2] models for the multiple scattering of ions transmitted through thin amorphous films. However, the assumption of amorphous targets - while, in most of the experiments, polycrystalline targets have been used-, is a significant limitation for the prediction of angular distributions [3] and for the use of multiple scattering (MS) models such as those from Refs. [1, 2]. At low energies where additional limitations for the application of MS models - such as the nonapplicability of the small angle approximation - appear, the availability of theoretical models is even more critical.

The aim of our work is to study the angular distribution of low-energy protons transmitted through thin gold films in an attempt to shed light on possible mechanisms responsible for the influence of the crystal structure on multiple scattering.

We have measured angular distributions of protons which have travelled through thin polycrystalline gold targets $(\sim 15 \mathrm{~nm})$ with incident energies in the range of $4-10 \mathrm{keV}$. The results confirm previous

\footnotetext{
"Corresponding author: e-mail: archubi@cab.cnea.gov.ar
} 
experiments at higher energies [3] showing deviations from the theoretical predictions based on the standard multiple scattering theory [1,2]. In order to prove that the effect of crystal structure is one of the main causes of these deviations we have performed numerical simulations which take into account the simultaneous interaction of the proton with nearest neighbours situated in the positions corresponding to a f.c.c. lattice.

To simulate the polycrystalline structure in a realistic way, we have made an analysis of the target by means of transmission electron microscopy (TEM) techniques. We observed that it was composed of microcrystals of a variety of sizes and irregular shapes $(5 \mathrm{~nm}$ to $40 \mathrm{~nm})$. That means that in a fraction of the target there is a superposition of two or more microcrystals. The most frequent are between $10 \mathrm{~nm}$ and $15 \mathrm{~nm}$. The microcrystals are randomly oriented and there is a significant fraction of twins $(30 \%$ to $40 \%$ ) inside the biggest ones. Including these characteristics in the simulation, together with the effect of thermal vibrations and crystal disorder produced by vacancies, interstitials, grain border and elastic strains, we analyzed the corresponding angular distributions.

The simulation results for the polycrystalline target suggest an important contribution of channeling effects in angular distributions. To understand the influence of the channelling effect, we have measured the angular distributions of $10 \mathrm{keV}$ protons transmitted through a thin monocrystalline gold target $(10 \mathrm{~nm})$ oriented in $\langle 100\rangle$ direction, and also for this case we made numerical simulations. As for the polycrystalline targets a TEM analysis was performed and a significant amount of small failures was found, but no twins were detected.

The simulation results are compared with the experiments, both for monocrystalline and polycrystalline gold foils, showing the critical influence of the target structure in the angular spectra of the transmitted beam.

\section{Experimental method and simulations}

The experimental arrangement has been described previously [4]. The angular analysis has been made rotating an electron multiplier detector with angular resolution of $1.9^{\circ}$ in the case of polycrystalline targets, and $0.5^{\circ}$ in the channeling experiment where an electrostatic analyzer preceded the detector. This analyzer allowed to filter out ions traversing pinholes present in the monocrystalline targets. When using this technique each point of the angular distribution was obtained integrating de corresponding energy spectrum.

Self-supported polycrystalline targets were elaborated by evaporation under clean vacuum conditions on a very smooth plastic substrate [5], which was subsequently dissolved. The foil thickness $(15.3 \mathrm{~nm})$ of the polycrystalline target was determined by matching proton energy-loss measurements to the stopping power tables of Andersen and Ziegler [6]. The smoothness of the foils was checked by the method described in Ref. [7]. The result was an estimated roughness of less than $12 \%$. The monocrystalline target used in the channeling experiments was a commercial one [8]. It had a thickness of $10 \mathrm{~nm}$. and it was oriented in the $\langle 100\rangle$ direction.

In the simulations the crystal structure is taken into account situating the ion in the central cell of a cluster of $3 \times 3 \times 3 \mathrm{fcc}$ cells. The resultant force of the interaction of the ion and all the surrounding atoms inside this cluster, up to a distance equivalent to one lattice constant, is derived from different types of screened Coulomb potentials. The trajectory of the ion is obtained by the discretization of the standard kinematic equations into time steps. Each time the ion exits the central cell of the cluster a new slab of $3 \times 3$ cells is generated in the corresponding direction. The backward slab of cells is eliminated and therefore the projectile is always in the central cell of a $3 \times 3 \times 3$ cluster.

The different starting points of the projectile when it hits the foil are simulated randomizing the initial position inside the central cell of the first cluster. In the case of a polycrystal the different random orientations of the microcrystals are simulated by randomizing the initial direction of the projectile relative to a coordinate system coincident with the principal axes of the crystal. When the ion travels through two or more overlapped microcrystals the direction of the new microcrystal is again simulated randomly changing the ion direction.

The angular distributions are obtained recording the emergence angle of each ion after a number of time steps corresponding to the target thickness. 


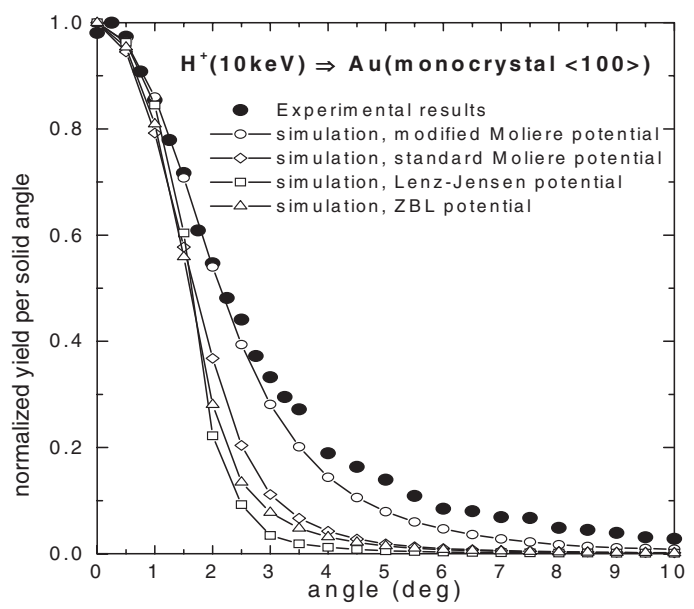

Fig. 1 Comparison of simulation with different potentials for protons in a $10 \mathrm{~nm}$ gold foil oriented in the $\langle 100\rangle$ direction. The Moliere screening length has been incremented by $80 \%$. The thermal vibration amplitude used is twice the corresponding to $300 \mathrm{~K}$.

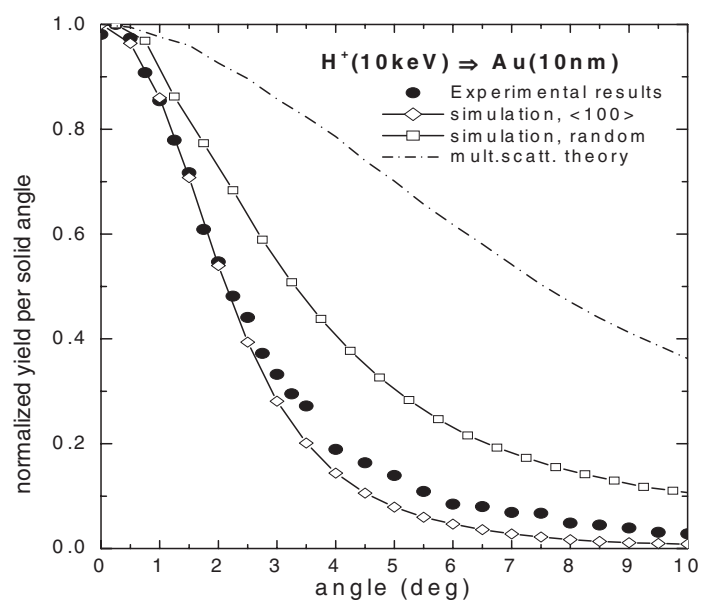

Fig. 2 Comparison between simulations for a $10 \mathrm{~nm}$ thick $\langle 100\rangle$ monocrystal layer, for a $10 \mathrm{~nm}$ layer with random orientated crystals and for an equivalent amorphous layer. The dashed line is a multiple scattering calculation with the formalism of Ref. [2] using a standard Moliere potential.

\section{Experimental results vs. simulation results}

\subsection{Channeling}

Figure 1 shows experimental angular distributions for channeled $10 \mathrm{keV}$ protons in a $10 \mathrm{~nm}$ monocrystalline gold foil oriented in the $\langle 100\rangle$ direction and the corresponding simulation results using several potentials. In order to compare in a realistic way we have convoluted the simulation results with the detector acceptance. The results show a reasonable agreement when using a Moliere potential whose screening distance is incremented by $80 \%$ and when increasing the thermal vibration amplitude by $100 \%$ respect to the corresponding to ambient temperature. We consider that this increment of the thermal vibration amplitude takes into account the influence of extra lattice disorder (grain borders, interstitials, vacancies, and other possible defects such as small failures in the crystal structure), but we consider this modification as tentative and subject to further investigation.

These results are in agreement with previous works where simulations has been performed by other methods $[9,10]$. However our simulations also showed that the influence of small initial angular deflections, small surface defects and a thin layer of light molecules adsorbed at the surfaces was negligible. We also evaluated the influence of electronic scattering including in the simulation an additional fluctuation in the angular dispersion due to scattering with target electrons according to the semiclassical approach used in Ref. [11]. The effect obtained in this way was found to be negligible. The simulation curves shown in Fig. 2 are the results after modifying the screening distance and the thermal vibration amplitude for a $\langle 100\rangle$ oriented crystal and for single layer, also $10 \mathrm{~nm}$ thick, of tiny crystals with random orientation. We note that there is a significant difference between the two curves. This figure also shows the multiple scattering function for an amorphous target of the same thickness. It yields a distribution considerably broader even than the corresponding to the random oriented tiny crystals. We assume that this is caused by ion channeling in those microcrystals which are adequately oriented.

In order to prove the consistence of our simulation results we also simulated the experiments performed by Machlin et al. [9] and obtained a very good agreement with their measurements modifying Moliere's screening distance and the thermal vibration amplitude in the same percentage we had used to simulate our experiments. 


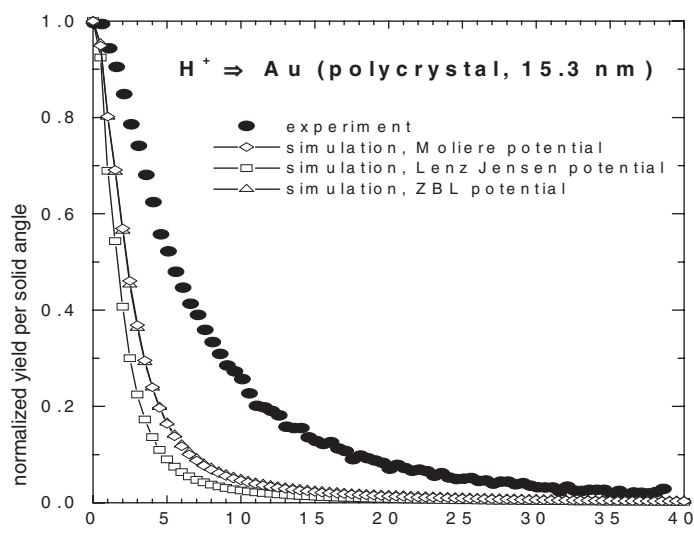

Fig. 3 Experimental angular distributions vs. simulation results for $10 \mathrm{keV}$ protons in a $15.3 \mathrm{~nm}$ polycrystalline gold foil using different standard potentials.

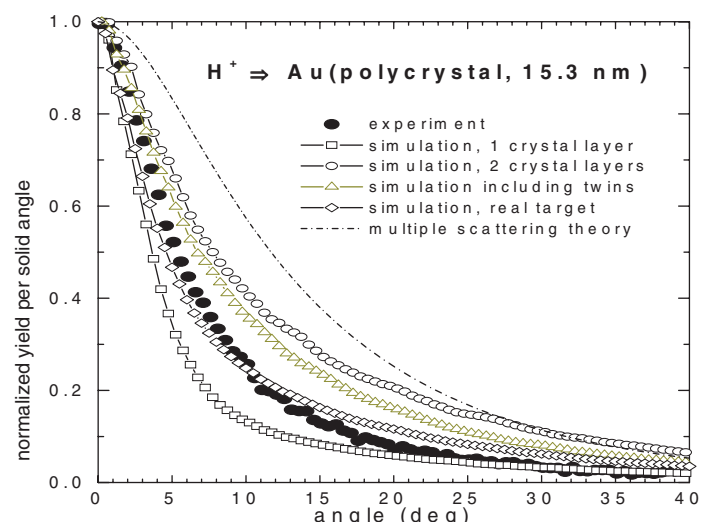

Fig. 4 Experiment vs. simulation results using a modified Moliere potential. The standard Moliere screening length has been incremented by $80 \%$. Thermal vibrations amplitude is twice the corresponding to $300 \mathrm{~K}$. The multiple scattering curve was calculated using the formalism of Ref. [2], with a standard Moliere potential.

\subsection{Polycrystalline target}

Figures 3 and 4 show the experimental results for $10 \mathrm{keV}$ protons traversing a $15.3 \mathrm{~nm}$ polycrystalline foil. To obtain a realistic representation of the polycrystalline target in the simulation we considered the microcrystal sizes and their appearance frequencies resulting from the TEM analysis. The target was considered as constituted by 3 sections and summed up the simulation results for each of them. One section, covering $30 \%$ of the area, constituted by microcrystals larger than $15 \mathrm{~nm}$, without defects; a second one, also covering $30 \%$, formed by microcrystals of the same size, however containing twin sequences; and the remaining section of $40 \%$ constituted by a superposition of 2 microcrystals of sizes between 5 and $15 \mathrm{~nm}$.

It can be observed that if standard potentials (Moliere, Lenz-Jensen, ZBL) are used no agreement with the experimental results are achieved even incrementing the thermal vibration (see Fig. 3), however, increasing the screening distance in Moliere's potential by the same percentages used for the channeling simulations, we obtain a good agreement as it is shown in Fig. 4.

\section{Conclusions}

We have confirmed the discrepancies between theoretical angular distribution for amorphous targets and measured angular distribution for a polycrystalline gold target observed at higher energies [3].

We interpret that angular distributions of protons traversing a gold polycrystalline target are influenced by the fraction of microcrystals oriented in channeling directions as well as by crystal imperfections.

The deterministic method we have used required modifications of the standard potentials to achieve an agreement with experimental results. In particular we have obtained the best results by modifying the screening distance of a Moliere potential. This way we have been able to reproduce the half-angle of the angular distributions in our experiments with polycrystalline and monocrystalline targets and also in other previous experiments performed by Machlin et al. [9].

Acknowledgement The support by the Argentine SECYT, grant PICT 03-03579, is acknowledged. 


\section{References}

[1] L. Meyer, phys. stat. sol. (b) 44, 253 (1971).

[2] P. Sigmund and K. Winterbon, Nucl. Instrum. Methods 119, 541(1974).

[3] H. H. Andersen, J. Bottiger, H. Knudsen, and P. Moller-Petersen, Phys. Rev. A 10, 1568 (1974).

[4] M. Famá, G. H. Lantschner, J. C. Eckardt, C. D. Denton, and N. R. Arista, Nucl. Instrum. Methods B 164/165, 241 (2000).

[5] A. Valenzuela and J. C. Eckardt, Rev. Sci. Instrum. 42, 127 (1971).

[6] H. H. Andersen and J. F. Ziegler, Stopping and Ranges of Ions in Matter, edited by J. F. Ziegler, Vol. 3 (Pergamon, New York, 1977).

[7] J. C. Eckardt and G. H. Lantschner, Thin Solid Films 249, 11 (1994).

[8] Pelco International, USA, www.pelcoint.com, Fax: 530-243-3761.

[9] E. S. Machlin and S. Petraglia, Nuovo Cimento B 55(1), 263 (1968).

E. S. Machlin et al., Phys. Lett. A 24, 173 (1970).

[10] R. Blume, PhD thesis, University Munich (1980).

[11] J. Lindhard, Mat. Fys. Medd. Dan. Vid. Selsk. 34, Nr 14 (1965). 